\title{
15.
}

\section{Allgemeine Betrachtung über einander doppelt berührende Kegelschnitte.}

(Von Herrn Professor Dr. J. Steiner zu Berlin.)

\section{\$. 10.}

An die vorhergehende Abhandlung, namentlich an diejenige Betrachtung, wo das Verhalten der gesammten Kegelschnitte, welche zwei feste Kreise doppelt berühren, angegeben worden, erlaube ich mir hier die etwas allgemeinere Betrachtung anzuschliefsen, wo statt der Kreise irgend zwei Kegelschnitte, die gleichfalls durch $\boldsymbol{A}^{2}$ und $\boldsymbol{B}^{2}$ bezeichnet werden mögen, in fester Lage gegeben sind, und wobei eben so die Eigenschaften aller sie doppelt berührenden Kegelschnitte berücksichtigt werden sollen.

I. Um einen bestimmten Fall (Figur) vor Augen zu haben, denke oder zeichne man zwei Ellipsen $\boldsymbol{A}^{2}$ und $\boldsymbol{B}^{2}$, welche einander in vier Puncten $r, s, t, u$ schneiden, und somit auch vier reelle gemeinschaftliche Tangenten $\boldsymbol{R}, \boldsymbol{S}, \boldsymbol{T}, \boldsymbol{U}$ haben; nämlich diejenige Tangente heifse $\boldsymbol{R}$, von deren Berührungspuncten aus zwei Bogen beider Ellipsen unmittelbar nach dem Schnitte $r$ führen; eben so die andern. Die vier Schnitte bilden ein vollständiges Viereck rstu und die vier Tangenten ein vollständiges Vierseit RSTU. In Betracht der drei Paar Gegenseiten des erstern und deren Schnitte, so wie in Rücksicht der drei Paar Gegenecken des letztern und dessen drei Diagonalen setze man:

$$
\begin{aligned}
& \text { Seite } \quad r s=\mathfrak{X} \text { und } \quad t u=\mathfrak{X}_{1} ; \quad \text { Schnitt } \mathfrak{X X}_{1}=x \text {. } \\
& \text { - } \quad r t=\mathfrak{Y} \text { und } \quad s u=\mathfrak{Y}_{1} ; \quad-\quad \mathfrak{Y Y Y}_{1}=y \text {. } \\
& \text { - } \quad r u=3 \text { und } s t=31 ; \quad-\quad 33_{1}=z \text {. }
\end{aligned}
$$

Ecke $R S=x$ und $T U=x_{1} ;$ Diagonale $x x_{1}=X$.

- $\boldsymbol{R T}=\mathfrak{y}$ und $\boldsymbol{S U}=\mathfrak{y}_{1} ; \quad-\quad \mathfrak{y}_{1}=\boldsymbol{Y}$.

- $\boldsymbol{R U}=\boldsymbol{z}$ und $\boldsymbol{S T}=z_{1} ; \quad-\quad z_{z_{1}}=\mathbf{Z}$.

Die Schnitte $x, y, z$ der drei Paar Gegenseiten des Vierecks sind das gemeinschaftliche Trippel conjugirte Pole und die drei Diagonalen $\boldsymbol{X}, \boldsymbol{Y}, \boldsymbol{Z}$ des Vierseits sind das gemeinschaftliche Trippel conjugirte Polaren der beiden Ellipsen, so dafs also auch. 


$$
\begin{aligned}
& \text { Schnitt } \boldsymbol{X} \dot{\boldsymbol{Y}}=\boldsymbol{z}, \quad \boldsymbol{X Z}=\boldsymbol{y}, \quad \boldsymbol{Y} \boldsymbol{Z}=\boldsymbol{x} \text { und } \\
& \text { Gerade } x y=Z, \quad x z=Y, \quad y z=X
\end{aligned}
$$

ist. Ferner sind dabei einerseits $x, z^{2}, y$ und $\mathfrak{z}_{1} ; x, \mathfrak{y}, z$ und $y_{1} ; y, x, z$ und $x_{1}$ vier harmonische Puncte, so wie andererseits $\boldsymbol{X}, \mathfrak{Z}, \boldsymbol{Y}$ und $\mathfrak{3}_{1} ; \boldsymbol{X}, \mathfrak{Y}, \boldsymbol{Z}$ und $\mathfrak{Y}_{1}$; $\boldsymbol{Y}, \mathfrak{X}, \boldsymbol{Z}$ und $\mathfrak{X}_{1}$ vier harmonische Gerade.

Mit Bezug hierauf und mit Berücksichtigung anderer, im vorigen Aufsatze bereits angewandter Bezeichnungen und Benennungen, lassen sich die erwähnten Eigenschaften, wie folgt, aussprechen.

II. Die gesammten Kegelschnitte $\boldsymbol{C}^{2}$, welche beide gegebenen Ellipsen $\boldsymbol{A}^{2}$ und $\boldsymbol{B}^{2}$ doppelt berühren, zerfallen vermöge ihrer Beziehung zu den drei Polen $x, y$ und $z$, in drei verschiedene Schaaren $\boldsymbol{S}\left(\boldsymbol{C}_{x}^{2}\right), \boldsymbol{S}\left(\boldsymbol{C}_{y}^{2}\right)$ und $\boldsymbol{S}\left(\boldsymbol{C}_{z}^{2}\right)$, (\$. 8.), welche sich jedoch im Allgemeinen gleich verhalten und gleiche Eigenschaften haben, so dafs wir Kürze halber nur von der einen Schaar, etwa von $\boldsymbol{S}\left(\boldsymbol{C}_{x}^{2}\right)$, zu sprechen brauchen.

1) „Berührt eine Curve $C_{x}^{2}$ die Ellipse $A^{2}$ in den Puncten $p$ und $\mu_{1}$, und die Ellipse $B^{2}$ in den Puncten $y$ und $y_{1}$, so gehen die Berührungssehnen $p p_{1}$ und $q q_{1}$ durch den Pol $x$ und sind allemal $z u$ den Gegenseiten $\mathfrak{X}$ und $\mathfrak{X}_{1}$ zugeordnet harmonisch." Und umgekehrt: „Zieht mun durch den Pol $x$ irgend swei su den Seiten $\mathfrak{X}$ und $\mathfrak{X}_{1}$ zugeordnete harmonische Gerude, etwa $G$ und $H$, so schneiden sie die Ellipsen $A^{2}$ und $\boldsymbol{B}^{2}$ besiehlich in solchen Puncten $p, p_{1}$ und $q, q_{1}$, in welchen dieselben von einer Curve $C_{x}^{2}$ berührt werden; und ferner schneiden sie verwechselt, $H$ die $A^{2}$ und $G$ die $B^{2}$ in solchen Puncten $p^{0}, p_{1}^{\prime \prime}$ und $q^{\prime \prime}, q_{1}^{\prime \prime}$, in welchen $A^{2}$ und $B^{2}$ von einer andern Curve $C_{x}^{2}$ berürt werden."

2) ,Werden zwischen je zwei Paar zusammengehörigen Berührungspuncten $p$ und $p_{1}, q$ und $q_{1}$ die vier Wechselsehnen $p q, p q_{1}, p_{1} q$ und $p_{1} q_{1}$ gezogen ( $(5.8 .5$.$) , so berühren dieselben insgesamnt einen be-$ stimmten Kegelschnitt, etwa $X^{2}$, welcher dem Vierseit RSTU eingeschrieben ist und auch die zwei Gegenseilen $\mathfrak{X}$ und $\mathfrak{X}_{1}$ berührt (indern die letztern, so wie die Tungenten $R, S, T, U$ specielle Wechselsehnen sind)." Und ferner: „Die aus den zwei Schnitten der Polare $\boldsymbol{X}$ mit der Ellipse $\boldsymbol{B}^{2}$ (oder $\boldsymbol{A}^{2}$ ) an den Kegelschnitt $\boldsymbol{X}^{2}$ gelegten zwei Paar Tangenten, berühren ihn in den nämlichen Puncten, in welchen er von der andern Ellipse $\boldsymbol{A}^{2}$ (oder $\boldsymbol{B}^{2}$ ) geschnitten wird."

3) ,Legt man durch die vier Berührungspuncte $p$ und $p_{1}, q$ und $q_{1}$ irgend einen willkürlichen Kegelschnitt $D^{2}$, so schneidet er die gege- 
214 15. J. Steiner, allgem. Betrachtung üb. cinund. doppelt berühr. Kegelschnitte.

benen Curven $A^{2}$ und $B^{2}$ in vier solchen neuen Puncten $p^{0}$ und $p_{1}^{0}, q^{\text {s }}$ und $q_{1}^{0}$, in welchen dieselben von einer andern Curve $C_{x}^{2}$ berührt werden." Und umgekehrt: „Die acht Berührungspuncte je zweier Curven $C_{x}^{2}$ mit den Ellipsen $A^{2}$ und $B^{2}$ liegen jedesmal in irgend einem Kegelschnitte $D^{2} . "-$ „Alle Curven $C_{x}^{2}$ haben gemeinschaftlich $x$ und $X$ zu Pol und Polaren. Von den gemeinschaftlichen Secunten je zweier Curven $C_{x}^{2}$ geht immer ein Paar, etwa $G$ und $H$, durch den Pol $x$ und sie sind allemal zu $\mathfrak{X}$ und $\mathfrak{X}_{1}$ zugeordnet harmonisch."

4) ,Jede vier Berührungspuncte $p, p_{1}, q, q_{1}$ liegen einerseits mit den Ecken $r$ und $s$ in einem Kegelschnitte, etwa $M^{2}$, und andererseits mit den Ecken $t$ und $u$ in einem Kegelschnitte $M_{1}^{2}$. Die gesammten hierdurch bestimmten Kegelschnitte $M^{2}$ berühren einander in den Puncten $r$ und $s$, so da/s sie daselbst gemeinschaftliche Berührungstangenten, etwa $\Re$ und $\mathfrak{S}$, haben, mit der gemeinschaftlichen Berührungssehne $r s=\mathfrak{X}$, und somit einen speciellen Curven-Büschel, $\boldsymbol{B}\left(\boldsymbol{M}^{2}\right)$, bilden. Der Schnitt der Tangenten $\Re$ und $\mathfrak{S}$ hei/se $m$; er liegt in der Polare $X$ und $m$ und $\mathfrak{X}$ sind Pol und Polare in Bezug auf alle $M^{2}$, auf $B\left(M^{2}\right)$. Seien a und $b$ die Pole der Seite $\mathfrak{X}$ in Bezug auf $\boldsymbol{A}^{2}$ und $\boldsymbol{B}^{2}$, dieselben liegen auch in $\boldsymbol{X}$, und sei $c$ der Schnitt von $X$ mit $\mathfrak{X}$ : so sind die vier Puncte $a, m, b, c$ harmonisch, so da/s also der Pol $m$ durch die, als gegeben anzusehenden drei Puncte $a, b, c$ bestimmt ist; und durch ihn sind dann auch die Tangenten $\Re$ und $\mathcal{S}(=m r$ und ms) bestimmt. Ganz eben so berühren alle Kegelschnitte $M_{1}^{2}$ einander in den Puncten $t$ und $u$, haben daselbst gemeinschaftliche Berührungstangenten $\mathfrak{T}$ und $\mathfrak{u}$, mit der Berührungssehne $t u=\mathfrak{X}_{1}$, und bilden einen speciellen Curven-Büschel $B\left(M_{1}^{2}\right)$; und ferner liegen der Schnitt $m_{1}$ von $\mathfrak{T}$ mit $\mathfrak{u}$, und die Pole $a_{1}$ und $b_{1}$ der Seite $\mathfrak{X}_{1}$ in Bezug auf $\dot{A}^{2}$ und $B^{2}$ in derselben Polare $X$, und ist zudem $c_{1}$ der Schnitt von $X$ mit $\mathfrak{X}_{1}$, so sind die vier Puncte $a_{1}, m_{1}, b_{1}$, c $c_{1}$ harmonisch, also durch $a_{1}, b_{1}$ und $c_{1}$ der Pol $m_{1}$ und durch ihn die Tangenten $\mathfrak{T}$ und $\mathfrak{u}$ bestimmt." - „Die auf diese Weise bestimmten zwei Paar Tangenten $\Re$ und $\mathfrak{S}, \mathfrak{I}$ und $\mathfrak{u}$ berühren auch den obigen Kegelschnitt $X^{2}$, den Ort aller Wechselsehnen (2.), und zwar berühren ihn $\Re$ und $\mathfrak{S}$ in ihren Schnitten mit der Seite $\mathfrak{X}_{1}$, und eben so berühren ihn $\mathfrak{I}$ und $\mathfrak{u}$ in ihren Schnitten mit der Seite $\mathfrak{X}$, so dafs also in Bezug auf $X^{2}$ verwechselt $m$ der Pol von $\mathfrak{X}_{1}$, und $m_{1}$ der Pol von $\mathfrak{X}$ ist." Werden diese zwei Paar Tangenten vorausgesetzt, so kann man auch umgekehrt be- 
15. J. St einer, allgem. Betrachtıng üb. einand. doppelt berühr. Kegelschnitte.

haupten: „Jeder Kegelschnitt $M^{2}$, velcher die Geraden $\Re$ und $\mathfrak{S}$ in den Puncten $r$ und $s$ berührt, schneidet die gegebenen Curven $A^{2}$ und $B^{2}$ in vier solchen Puncten $p$ und $p_{1}, q$ und $q_{1}$ (au/ser in $r$ und s), in welchen sie von einer Curve $C_{x}^{2}$ berührt werden." „Die gegenseitigen vier Schnitte je zweier Curven $C_{x}^{2}$ liegen allemal in einem Kegelschnitte $M^{2}$ (der $\Re$ und $\mathfrak{S}$ in $r$ und $s$ berührt); und umgekehrt: jeder Kegelschnitt $M^{2}$ schneidet jede Curve $C_{x}^{2}$ in solchen vier Puncten, durch welche allemal noch irgend eine andere Curve $C_{x}^{2}$ geht." Gleiches gilt für die Kegelschnitte $M_{1}^{2}$.

III. 1) ,Sind $P$ und $\boldsymbol{P}_{1}, Q$ und $Q_{1}$ die Berührungstangenten einer $C_{x}^{2}$ mit den gégebenen Curven $A^{2}$ und $B^{2}$, so liegen die Schnitte $\boldsymbol{P P}_{1}=p$ und $\boldsymbol{Q Q} Q_{1}=p_{1}$ stets in der Polare $\boldsymbol{X}$ und sind allemal ou den Ecken $\mathfrak{x}$ und $\mathfrak{x}_{1}$ zugeordnet harmonisch." Und umgekehrt: „Nimmt man in der Polare $X$ irgend zwei zu den Gegenecken $x$ und $x_{1}$ zugeordnete harmonische Puncte, etwa $g$ und $h$, an, so sind die aus ihnen an die Curven $A^{2}$ und $B^{2}$ gezogenen Tangenten $P$ und $P_{1}, Q$ und $Q_{1}$ zugleich die Berührungstangenten dieser Curven mit irgend einer Curve $C_{x}^{2}$; und eben so sind die (verwechselt) aus $h$ und $g$ beziehlich an $A^{2}$ und $B^{2}$ gelegten Tangenten $\boldsymbol{P}^{0}$ und $\boldsymbol{P}_{1}^{0}, \boldsymbol{Q}^{0}$ und $\boldsymbol{Q}_{1}^{0}$ zugleich die Berührungstangenten einer $C_{x}^{2}$ mit $A^{2}$ und $B^{2} . "$

2) „Jede zwei Paar zusammengehörige Berührungstangenten $P$ und $P_{1}, Q$ und $Q_{1}$ haben vier Wechselschnitte $P Q$, und $P Q_{1}, P_{1} Q$ und $P_{1} Q_{1} ;$ der Ort aller dieser Wechselschnitte ist ein bestimmter Kegelschnitt, etua $\mathfrak{X}^{2}$, welcher dem Viereck rstu ungeschrieben ist, und zudem durch die Gegenecken $x$ und $x_{1}$ geht." Und ferner: „Die aus dem Pol $x$ an die Ellipse $\boldsymbol{B}^{2}$ (oder $\boldsymbol{A}^{2}$ ) gezogenen Tangenten schneiden den Kegelschnitt $\mathfrak{X}^{2}$ in denselben Puncten, in denen er von den vier Tangenten berührt wird, welche er mit der andern Ellipse $A^{2}$ (oder $B^{2}$ ) gemein hat."

3) „Werden die vier Berührungstangenten $P$ und $P_{1}, Q$ und $Q_{1}$ von einem willkürlichen andern Kegelschnitte $\boldsymbol{D}^{2}$ berührt, so hat dieser mit den gegebenen Curven $\boldsymbol{A}^{2}$ und $\boldsymbol{B}^{2}$ noch zwei neue Paare Tangenten $\boldsymbol{P}^{0}$ und $\boldsymbol{P}_{1}^{0}, \boldsymbol{Q}^{0}$ und $\boldsymbol{Q}_{1}^{0}$ gemein, welche allemal die Berührungstangenten einer andern Curve $C_{x}^{2}$ mit $A^{2}$ und $B^{2}$ sind." Und umgekehrt: „Die acht Berührungstangenten irgend zweier Curven $C_{x}^{2}$ mit den Curven $A^{2}$ und $B^{2}$ werden allemal von irgend einem Kegelschnitte $D^{2}$ berührt." - „Von den gegenseiligen Schnitten der gemeinschaftlichen Tangenten je zweier 28 * 
216 15. J. St einer, allgem. Betrachtung üb. einand. doppelt berühr. Kegelschnitte.

Curven $C_{x}^{2}$ liegt ionmer ein Paar, etwa $g$ und $h$, auf der Polare $X$ und sie sind allemal $z u$ den Ecken $x$ und $x_{1}$ sugeordnet harmonisch."

4) „Jede vier Berührungstangenten $P, P_{1}, Q, Q_{1}$ werden einerseits mit den Tangenten $\boldsymbol{R}$ und $\boldsymbol{S}$ susummen von einem Kegelschnitte $\mathfrak{M}^{2}$, und andererseits mit den Tangenten $T$. und $U$ susammen von einem Kegelschnitte $\mathfrak{M}_{1}^{2}$ berührt. Alle hierdurch bestimmten Kegelschnilte $\mathfrak{M}^{2}$ berühren die Tangenten $R$ und $\boldsymbol{S}$ in den nümlichen Puncten, etwa $\mathfrak{r}$ und $\mathfrak{s}$, und somit auch einander selbst, so da/s sie $\mathfrak{r}=\mathfrak{M}$ sur gemeinschaftlichen Berührungssehne, so wie $x$ und $\mathfrak{M}$ gemeinschaftlich zu Pol und Polare haben, und einen speciellen Curven-Büschel, $\boldsymbol{B}\left(\mathfrak{M}^{2}\right)$ bilden. Die Berührungssehne $\mathfrak{M}$ geht durch den Pol $x$; eben so die Polaren von $x$ in Bezug auf $A^{2}$ und $B^{2}$, die $\mathfrak{A}$ und $\mathfrak{B}$ heifsen mögen; und wird noch die Gerade $x \mathfrak{x}=\mathfrak{C}$ gesetzt, so sind die vier Geraden $\mathfrak{A}, \mathfrak{A}, \mathfrak{B}$, $\mathfrak{C}$ harmonisch; somit ist $\mathfrak{M}$ durch die drei übrigen, die als gegeben anzusehen sind, bestimmt, und durch $\mathfrak{M}$ sind dann uuch die Puncte $\mathfrak{x}$ und $\mathfrak{s}$ bestimmt, als ihre Schnitte mit $R$ und $S$. Ganz eben so verhält es sich mit den Kegelschnitten $\boldsymbol{M}_{1}^{2}$, mit $\boldsymbol{B}\left(M_{1}^{2}\right)$, welche die Tangenten $\boldsymbol{T}$ und $\boldsymbol{U}$ gleicherweise in zwei bestimmten Puncten $\mathfrak{t}$ und $\mathfrak{u}$ berühren, $u$. s. w." „Die auf diese Weise bestimmten zwei Paar Berührungspuncte $\mathfrak{r}$ und $\mathfrak{t}$ und $\mathfrak{u}$ liegen in dem obigen Kegelschnitte $\mathfrak{X}^{2}$, dem Ort der Wechselschnitle (2.), und zwar gehen die in $\mathfrak{x}$, an $\mathfrak{X}^{2}$ gelegten Tangenten beide durch die Ecke $x$, und die in $t, \mathfrak{u}$ an densèlben gelegten Tangenten beide durch die Ecke $x_{1}$, so da/s also in Bezuy auf $\mathfrak{X}^{2}$ verkehrt $\mathfrak{M}$ die Polare von $\mathfrak{x}_{1}$, und $\mathfrak{M}_{1}$ die Polare von $\mathfrak{x}$ ist." Bei Voraussetzung der vier Puncte $\mathfrak{r}$ und $\mathfrak{s}, \mathfrak{t}$ und $\mathfrak{a}$ kann man umgekehrt sagen: „Jeder Kegelschnilt $\mathfrak{M}^{2}$ (oder $\mathfrak{M}_{1}^{2}$ ), welcher die Tangenten $R$ und $S$ (oder $T$ und $U$ ) in den Puncten $\mathfrak{r}$ und $\mathfrak{g}$ (oder $t$ und $\mathfrak{u}$ ) berultrt, hat mit den gegebenen Curven $A^{2}$ und $B^{2}$, au/ser jenen Tangenten noch zwei solche Paare Tangenten gemein, $\boldsymbol{P}$ und $\boldsymbol{P}_{1}, \boldsymbol{Q}$ und $\boldsymbol{Q}_{1}$, welche sugleich die Berührungstangenten derselben mit einer Curve $C_{x}^{2}$ sind." Und ferner: „Die gemeinschaftlichen Tangenten je zweier Curven $C_{x}^{2}$ berühren allemal sugleich einen der Kegelschnitte $\mathfrak{M}^{2}$ sowohl, als auch einen der Kegelschnitle $\mathfrak{M}_{1}^{2}$; und umgekehrt: jeder Kegelschnitt $\mathfrak{M}^{2}$ oder $\mathfrak{M}_{1}^{2}$ hat mit jeder Curve $C_{x}^{2}$ solche vier Tangenten gemein, welche allemal auch noch von einer andern Curve $C_{x}^{2}$ berührt werden." 
15. J. St einer, allgem. Betrachtung üb: einand.doppelt berïhr. Kegelschnitte. 217

IV. „Legt man an jede Curve $C_{x}^{2}$, in ihren beiden Schnitten mit der Seite $\mathfrak{Y}$ (I.), die Tangenten, so geht ron diesen zwei Tangenten stets die eine durch die Ecke $z$ und die andere durch die Ecke $z_{1}$; und eben so gehl von den zwei Tangenten derselben Curve $C_{x}^{2}$, in ihren Schnitten mit der Seite $\mathfrak{Y}_{1}$, iinmer die eine durch $z$ und die andere durch $z_{1} . "$ Oder umgekehrt: „Zieht man aus der Ecke o oder jo an eine Curve $C_{x}^{2}$ die beiden Tangenten, so liegt allemal der Berührungspunct der einen Tangente in der Seile $\mathfrak{Y}$ und der Berührungspunct der andern in der Seite $\mathfrak{Y}_{1} . "$ - ,Und gleicherweise geht von den zwei Tanyenten jeder Curve $C_{x}^{2}$, in ihren Schnitten mit der Seite 3 oder $3_{1}$, allemal die eine durch die Ecke $y$ und die undere durch die Ecke $y_{1}$; oder ungekehrt: von den Berührungspuncten der aus der Ecke $y^{\prime}$ und $y_{1}$ an jede Curve $C_{x}^{2}$ gezogenen swei Tangenten, liegt der eine in der Seite 3 und der undere in der Seite $3_{1} . "$

Alle vorstehenden, sich auf die $\mathbf{S}\left(\boldsymbol{C}_{x}^{2}\right)$ allein beziehenden Sätze finden analogerweise, wie schon bemerkt worden, auch für die beiden andern Schaaren, $\boldsymbol{S}\left(\boldsymbol{C}_{y}^{2}\right)$ und $\boldsymbol{S}\left(\boldsymbol{C}_{z}^{2}\right)$, statt, so dafs also jeder Satz dreifach vorhanden ist. Die jedesmaligen zusammengehörigen Elemente sind leicht zu erkennen. Z. B. beim Satze (IV.), wo ungleichnamige Elemente zusammengehören, ist die Verbindung:

$$
\boldsymbol{S}\left(\boldsymbol{C}_{x}^{2}\right) \text { mit }\left\{\begin{array}{llll}
\mathfrak{Y}, & \mathfrak{Y}_{1} \text { und } \mathfrak{z}, & \mathfrak{z}_{1} \\
\mathfrak{Z}, & \mathfrak{Z}_{1} \text { und } \mathfrak{y}_{1}, \mathfrak{y}_{1} ;
\end{array}\right\}
$$

und danach ist die Verbindung für die beiden andern Fälle:

$$
\boldsymbol{S}\left(\boldsymbol{C}_{y}^{2}\right) \text { mit }\left\{\begin{array}{llll}
\mathfrak{X}, & \mathfrak{X}_{1} \text { und } \mathfrak{z}, \mathfrak{z}_{1} ; \\
\mathfrak{Z}, & \mathfrak{Z}_{1} \text { und } x, x_{1} ;
\end{array}\right\} ; \text { und } \boldsymbol{S}\left(\boldsymbol{C}_{z}^{2}\right) \text { mit }\left\{\begin{array}{lll}
\mathfrak{X}, & \mathfrak{X}_{1} \text { und } \mathfrak{y}, \mathfrak{y}_{1} ; \\
\mathfrak{Y}, \mathfrak{Y}_{1} \text { und } \mathfrak{x}, \mathfrak{x}_{1} \cdot
\end{array}\right\}
$$

Es giebt aber auch Sätze, welche sich auf zwei Schaaren zugleich beziehen, wie z. B. die folgenden.

V. 1) ,Alle Pole der Seile $\mathfrak{X}$ in Bezug auf die $\boldsymbol{S}\left(C_{y}^{2}\right)$ sowohl als in Bezug auf die $\boldsymbol{S}\left(C_{z}^{2}\right)$, nebst ihren beiden Polen a und $b$ in Bezug auf $\boldsymbol{A}^{2}$ und $\boldsymbol{B}^{2}$, liegen insgesammt in einem und demselben Kegelschnitle $\boldsymbol{M}_{x}^{2}$, welcher mit sum obigen Büschel $\boldsymbol{B}\left(\boldsymbol{M}^{2}\right)$ (II. 4.) gehört, daher durch die Ecken $r$ und $s$ geht und daselbst die Geraden $\Re$ und $\mathfrak{S}$ berührt, und

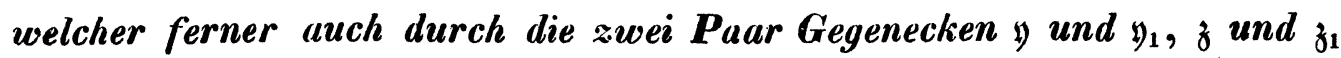
des Vierseits RSTU geht." „Gleicherweise liegen die gesammtén Pole der Seite $\mathfrak{F}_{1}$ in $B$ esug auf $\boldsymbol{S}_{(}\left(C_{y}^{2}\right)$ und $\boldsymbol{S}\left(C_{z}^{2}\right)$, nebst ihren Polen $a_{1}$ und $b_{1}$ in Bezug auf $A^{2}$ und $B^{2}$, in einem Kegelschnitte $M_{1 x}^{2}$, welcher zum obigen $\boldsymbol{B}\left(\boldsymbol{M}_{1}^{2}\right)$ (II. 4.) gehört, daher durch die Ecken $t$ und u geht und daselbst 
15. J. St einer, allgem. Betrachtung üb. einand. doppelt berïhr. Kegclschnitte.

die $\mathfrak{T}$ und $\mathfrak{d}$ berührt, und welcher ferner auch durch die nämlichen Gegenecken $y$ und $\mathfrak{y}_{1}, z$ und $z_{1}$ geht." - Auch dieser Satz findet dreifach statt; nämlich in Rücksicht auf jedes der drei Paar Gegenseiten des Vierecks rstu und der beiden mit dem jedesmaligen Paar ungleichnamigen Schaaren.

2) „Alle Polaren der Ecke $x$ in Bezug auf $\boldsymbol{S}\left(C_{y}^{2}\right)$ und $\mathbf{S}\left(C_{z}^{2}\right)$, nebst ihren Polaren $\mathfrak{A}$ und $\mathfrak{B}$ in Bezug auf $\boldsymbol{A}^{2}$ und $B^{2}$, berühren insgesammt einen bestimmten Kegelschnitt $\mathfrak{M}_{x}^{2}$, welcher $\mathbf{z u m}$ obigen $\boldsymbol{B}\left(\mathfrak{M}^{2}\right)$ (III. 4.) gehört und duher die Tangenten $R$ und $S$ in den Puncten $\mathfrak{r}$ und $\mathfrak{s}$ berührt, und welcher ferner auch die zwei Paar Gegenseiten $\mathfrak{Y}$ und $\mathfrak{Y}_{1}, 3$ und $\mathfrak{Z}_{1}$ des Vierecks $r$ stu zu Tangenten hat." „Und gleicherweise berühren alle Polaren der Ecke $x_{1}$ in Bezug auf $\boldsymbol{S}\left(C_{y}^{2}\right)$ und $\boldsymbol{S}\left(\boldsymbol{C}_{z}^{2}\right)$, nebst ihren Polaren $\mathfrak{A}_{1}$ und $\mathfrak{B}_{1}$ in Bezug auf $\boldsymbol{A}^{2}$ und $\boldsymbol{B}^{2}$, einen Kegelschnitt $\mathfrak{M}_{1 x}^{2}$, welcher zum obigen $\boldsymbol{B}\left(\mathfrak{M}_{1}^{2}\right)$ gehört, und daher die Tangenten $\boldsymbol{T}$ und $\boldsymbol{U}$ in den bestimmten Puncten $\mathfrak{t}$ und $\mathfrak{t}$, so wie ferner auch die Seiten $\mathfrak{Y}$ und $\mathfrak{Y}_{1}, 3$ und $\mathfrak{Z}_{1}$ berührt."

VI. Bemerkung. Die angegebenen Eigenschaften gelten für den vorausgesetzten Fall, dafs sowohl die vier gegenseitigen Schnitte als die vier gemeinschaftlichen Tangenten der gegebenen Kegelschnitte $\boldsymbol{A}^{2}$ und $\boldsymbol{B}^{2}$ reell sind, wobei jedoch die letztern nicht gerade Ellipsen sein müssen, sondern von beliebiger Art sein können. Von diesem Falle aus kann man zu den übrigen Fällen übergehen, bei welchen jo ein Theil der genannten Elemente imaginär wird. Die wesentlichsten Fälle der Art sind folgende drei. Wenn die gegenseitige Lage der gegebenen, beliebigen Kegelschnitte $A^{2}$ und $B^{3}$ so beschaffen ist, dafs entweder: 1) nur die vier Schnitte $r, s, t$ und $u$ reell, dagegen die vier gemeinschaftlichen Tangenten imaginär; oder 2) nur die vier gemeinschaftlichen Tangenten reell, dagegen die vier Schnitte imaginär; oder endlich 3) nur zwei Schnitte und nur zwei gemeinschaftliche Tangenten reell sind. Bei diesen drei Fällen wird dann auch von den übrigen, oben beschriebenen (I.) Elementen je ein Theil imaginăr, wodurch in den angegebenen Eigenschaften und Sätzen entsprechende, wenig oder mehr erhebliche Änderungen eintreten; ähnlich wie oben \$. 8. So tritt z. B., wenn etwa die Gegenseiten $\mathfrak{X}$ und $\mathfrak{X}_{1}$ imaginär werden, aber ihr Schnitt, der Pol $x$, reell bleibt, bei dem Satze (II. 1.) die Änderung ein, dafs die sämmtlichen Paare Berührungssehnen $p p_{1}$ und $q q_{1}$ ein elliptisches Strahlen-System bilden, wogegen sie dort ein hyperbolisches bilden. U. s. w. 
15. J. St einer, allgem. Betrachtung üb. einand. doppelt berïhr. Kegelschnitte.

\section{\$. 11.}

I. In Rücksicht der vorstehenden allgemeinen Sätze (\$. 10.) sollen hier noch folgende, in denselben mit inbegriffene, specielle Sätze besonders herausgehoben werden.

1) ,Werden einem vollständigen Vierseit RSTU irgend zwei Kegelschnitte $\boldsymbol{A}^{2}$ und $\boldsymbol{B}^{2}$ eingeschrieben, so liegen die 8 Puncte, in welchen sie die Seiten berühren, allemal in irgend einem dritten Kegelschnitte $D^{2} . "$ Und: „Legt man durch die vier Puncte $\mathfrak{x}, \mathfrak{b}, \mathfrak{t}$ und $\mathfrak{u}$, in welchen ein beliebiger Kegelschnitt $A^{2}$ die Seiten $R, S, T$ und $U$ des Vierseits berührt, einen willkürlichen Kegelschnitt $\boldsymbol{D}^{2}$, so schneidet dieser die Seiten in vier solchen neuen Puncten $\mathfrak{r}_{1}, \mathfrak{B}_{1}, \mathfrak{t}_{1}$ und $\mathfrak{u}_{1}$, in welchen dieselben allemal von irgend einern Kegelschnitte $\boldsymbol{B}^{2}$ berührt uerden." *) Ferner: „Die gegenseitigen vier Schnitte $r, s, t, u$ je zweier demselben Vierseit RSTU eingeschriebenen Kegelschnitte $A^{2}$ und $B^{2}$ liegen mit jedem Paar Gegenecken des Vierseits, also sowohl mit $x$ und $\mathfrak{x}_{1}$, als y) und $\mathfrak{y}_{1}$, und $z$ und $z_{1}$, zusammen in einem Kegelschnitte, beziehlich $\mathfrak{X}^{2}, \mathfrak{Y}^{2}$ und $\mathfrak{3}^{2}$." Und ferner: „Von den 8 Berührungspuncten $\left(\mathfrak{r}, \mathfrak{s}, \mathfrak{t}, \mathfrak{u} ; \mathfrak{r}_{1}, \mathfrak{g}_{1}, \mathfrak{t}_{1}, \mathfrak{u}_{1}\right)$ je zweier dem Vierseit eingeschriebenen Kegelschnitte $\boldsymbol{A}^{2}$ und $\boldsymbol{B}^{2}$ liegen 12 mal 4 mit irgend zwei der vier Schnitte $r, s, t, u$ der letztern zusammen in einem Kegelschnilte $M^{2}$ (oder $M_{1}^{2}$ ), und diese 12 Kegelschnitte ordnen sich in 6 Paare, welche einander doppelt berühren; nümlich durch je zwei der vier Schnitte $r, s, t, u$ gehen zwei Kegelschnitte $M^{2}$ und berühren sich in denselben. Die je 6 Puncte, welche zusammen in einem Kegelschnitte $M^{2}$ liegen, sind:

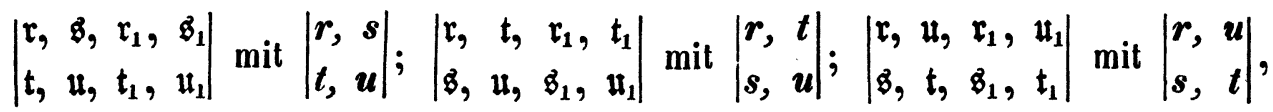

d.h. beide vier Puncte in der ersten Klammer liegen mit jedem Paar in der zueiten Klammer in einem $M^{2}$."

2) „Werden einem Viereck rstu zwei beliebige Kegelschnitte $A^{2}$ und $\boldsymbol{B}^{2}$ umgeschrieben und in den vier Ecken an dieselben Tangenten gelegt, so berühren die 8 Tangenten allemal irgend einen dritten Kegelschnitt $D^{2}$." Und: „Ist dem Viereck ein beliebiger Kegelschnitt $A^{2}$ umgeschrieben und werden dessen Tangenten $\Re, \mathfrak{S}, \mathfrak{I}$ und $\mathfrak{H}$, in den Ecken $r, s, t$ und $u$ des Vierecks, von einem willkürlichen Kegelschnitte $D^{2}$ be-

*) Die diesen zwei Sätzen analogen Sätze in Bezug auf das Dreiseit habe ich schon früher, 1828, in den Gergonne'schen Annales des Mathem. $t$. XIX. oder XX. bewiesen. 
rührt, so gehen aus den Ecken an den letztern noch vier solche neue Tangenten $\mathfrak{R}_{1}, \mathfrak{S}_{1}, \mathfrak{T}_{1}$ und $\mathfrak{u}_{1}$, welche in den Ecken allernal von irgend einem Kegelschnitte $\boldsymbol{B}^{2}$ berührt werden." Ferner: ,Die vier gemeinschaftlichen Tangenten $R, S, T, U$ je zueier demselben Viereck rstu umgeschriebenen Kegelschnitte $\boldsymbol{A}^{2}$ und $\boldsymbol{B}^{2}$ werden mit jedem der drei Paur Gegenseiten des Vierecks, (mit $\mathfrak{X}$ und $\mathfrak{X}_{1}, \mathfrak{Y}$ und $\mathfrak{Y}_{1}, 3$ und $\mathfrak{Z}_{1}$ ), zusammen von einem Kegelschnilte $\left(\boldsymbol{X}^{2}, \boldsymbol{Y}^{2}, \mathbf{Z}^{2}\right)$ berührt." Und ferner: „Bei je zuiei dem Viereck rstu umgeschriebenen Kegelschnitten $A^{2}$ und $B^{2}$ werden von ihren 8 Tangenten $\left(\Re, \mathfrak{S}, \mathfrak{T}, \mathfrak{u} ; \Re_{1}, \mathfrak{S}_{1}, \mathfrak{I}_{1}, \mathfrak{u}_{1}\right)$ in den Ecken 12 mal 4 mit irgend swei ihrer 4 gemeinschaflichen Tangenten $(\boldsymbol{R}, \boldsymbol{S}, \boldsymbol{T}, \boldsymbol{U})^{\prime}$ zusammen von irgend einem Kegelschnitte $\mathfrak{M}^{2}$ berührt; und swar ordnen sich diese 12 Kegelschnitte $\mathfrak{M}^{2}$ in 6 einander doppelt berührende Paare, welche die vier gemeinschaftlichen Tangenten $\boldsymbol{R}, \mathbb{S}$, $T$ und $U$, paarweise genommen, zu Berührungstangenten haben."

II. Von der obigen Betrachtung ( $\$$. 10.) kann man auch zu denjenigen besondern Fällen übergehen, wobei die gegebenen Kegelschnitte $\boldsymbol{A}^{2}$ und $\boldsymbol{B}^{2}$, einzeln genommen, aus einem Paar Puncten oder Geraden bestehen. In dieser Hinsicht sind folgende fünf Fälle zu beachten.

1) Wenn etwa $\boldsymbol{B}^{2}$ aus zwei Geraden 3 und $3_{1}$ besteht, so sind diese ein Paar Gegenseiten des Vierecks rstu, und ihr gegenseitiger Schnitt ist der Pol z. Die vier gemeinschaftlichen Tangenten $\boldsymbol{R}, \boldsymbol{S}, \boldsymbol{T}$ und $\boldsymbol{U}$ fallen paarweise zusammen, $(\boldsymbol{R U})$ und $(\boldsymbol{S T})$, und sind die aus dem Pol $z$ an die Curve $\boldsymbol{A}^{2}$ gehenden zwei Tangenten. Dadurch vereinigen sich von den sechs Ecken des früheren Vierseits $\boldsymbol{R S T U}$ zwei Paar, nämlich $\mathfrak{x}$ und $\mathfrak{x}_{1}, \mathfrak{y}$ und $\mathfrak{y}_{1}$ mit dem Puncte $z$, und die zwei übrigen, $z$ und $z_{1}$, sind die Berührungspuncte jener Tangenten $(\boldsymbol{R U})$ und $(\boldsymbol{S T})$, liegen in der Polare $\boldsymbol{Z}$ und sind zu den Polen $x$ und $y$ zugeordnet harmonisch. Hierbei artet die $\boldsymbol{S}\left(\boldsymbol{C}_{z}^{2}\right)$ in einen Strahlbüschel um den Mittelpunct $z$ aus, d. h. jeder durch $\boldsymbol{z}$ gehende Strahl (Gerade), doppelt gedacht, ist als eine $C_{z}^{2}$ anzusehen, seine Schnitte mit $\boldsymbol{A}^{2}$ sind zugleich seine Berührungspuncte' mit $\boldsymbol{A}^{2}$, wogegen seine $\mathrm{Be}-$ rührungspuncte mit $\boldsymbol{B}^{2}=\left(33_{1}\right)$ in $z$ vereinigt liegen. Die Schaaren $\boldsymbol{S}\left(\boldsymbol{C}_{x}^{2}\right)$ und $\boldsymbol{S}\left(\boldsymbol{C}_{y}^{2}\right)$ bleiben eigentliche Curven und behalten ihre obigen Eigenschaften, jedoch zum Theil mit angemessenen Modificationen.

2) Wenn $\boldsymbol{B}^{2}$ aus zwei Puncten $z$ und $z_{1}$ besteht, so sind diese ein Paar Gegenecken des Vierseits RSTU und liegen in der Polare $\boldsymbol{Z}$. Die vier Schnitte $r, s, t$ und $u$ rũcken paarweise zusammen, $(r u)$ und $(s t)$, in 
die Schnitte von $z_{z_{1}}=\boldsymbol{Z}$ mit der Curve $\boldsymbol{A}^{2}$, so dafs zwei Paar Gegenseiten des Vierecks rstu, nämlich $\mathfrak{X}$ und $\mathfrak{X}_{1}, \mathfrak{Y}$ und $\mathfrak{Y}_{1}$, auf $\mathbf{Z}$ fallen, und das dritte Paar, 3 und $3_{1}$, die Tangenten an $A^{2}$ in jenen Puncten $(r u)$ und $(s t)$ werden, einander in $z$ schneiden und zu den Polaren $\boldsymbol{X}$ und $\boldsymbol{Y}$ zugeordnet harmonisch sind. Hier besteht die $\boldsymbol{S}\left(\boldsymbol{C}_{z}^{2}\right)$ aus allen Paaren Tangenten der Curve $\boldsymbol{A}^{2}$, welche sich auf der Geraden $\boldsymbol{Z}$ schneiden. Dagegen enthalten $\boldsymbol{S}\left(\boldsymbol{C}_{x}^{2}\right)$ und $\boldsymbol{S}\left(\boldsymbol{C}_{y}^{2}\right)$ alle eigentlichen Kegelschnitte $\boldsymbol{C}^{2}$, welche durch die gegebenen Puncte $z$, $z_{1}$ gehén und die gegebene Curve $\boldsymbol{A}^{2}$ doppelt berühren.

3) Wenn $\boldsymbol{A}^{2}$ und $\boldsymbol{B}^{2}$ aus zwei Paar Geraden $\mathfrak{Y}$ und $\mathfrak{Y}_{1}, 3$ und $\mathfrak{Z}_{1}$ bestehen, so sind sie als zwèi Paar Gegenseiten des Vierecks $r$ stu anzusehen, ihre eigenen Schnitte als die Pole $y$ und $z$, ihre Wechselschnitte als die Schnitte $r, s, t$ und $u$. Die vier gemeinschaftlichen Tangenten $\boldsymbol{R}, \boldsymbol{S}$, $\boldsymbol{T}$ und $\boldsymbol{U}$ fallen alle auf die Gerade $\boldsymbol{y} z=\boldsymbol{X}$. Die Schaaren $\boldsymbol{S}\left(\boldsymbol{C}_{y}^{2}\right)$ and $\boldsymbol{S}\left(\boldsymbol{C}_{z}^{2}\right)$ arten in Strahlbüschel um die Pole $\boldsymbol{y}$ und $\approx$ aus, in gleichem Sinne wie oben (1.), und es bleibt nur die $\boldsymbol{S}\left(\boldsymbol{C}_{x}^{2}\right)$ als eigentliche Curven übrig, deren Berührungssehnen durch den $\mathrm{Pol} x$, deren Wechselsehnen dagegen parweise durch die Pole $y$ und $z$ gehen ( $\$$. 10. II.).

4) Wenn $\boldsymbol{A}^{2}$ und $\boldsymbol{B}^{2}$ aus zwei Paar Puncten $y$ und $y_{1}, z$ und $z_{1}$ bestehen, so sind sie als Gegenecken des Vierseits RSTU anzusehen, die sie verbindenden Geraden $y y_{1}$ und $z_{z_{1}}$ als die Polaren $\boldsymbol{Y}$ und $\boldsymbol{Z}$, und die beide Paare wechselseitig verbindenden Geraden als die gemeinschaftlichen Tangenten $\boldsymbol{R}, \boldsymbol{S}, \boldsymbol{T}$ und $\boldsymbol{U}$. Die vier Schnitte $r, s, t$ und $\boldsymbol{u}$ liegen alle im Pol $\boldsymbol{x}=\boldsymbol{Y}^{\prime} \boldsymbol{Z}$ vereint. Die $\boldsymbol{S}\left(\boldsymbol{C}_{y}^{2}\right)$ artet aus in alle Paare Gerade, welche durch die Puncte $z$ und $z_{1}$ gehen und sich auf $\boldsymbol{Y}$ schneiden; und eben so besteht die $\boldsymbol{S}\left(\boldsymbol{C}_{z}^{2}\right)$ aus allen Paaren Gerade, welche durch $y$ und $y_{1}$ gehen und sich auf $\boldsymbol{Z}$ schneiden. Die $\boldsymbol{S}\left(\boldsymbol{C}_{x}^{2}\right)$ bleiben eigentliche Curven, die dem Viereck $y y_{1} z_{z_{1}}$ umgeschrieben sind.

5) Wenn endlich $\boldsymbol{A}^{2}$ aus zwei Puncten $z_{\text {und }} z_{1}$, und $\boldsymbol{B}^{2}$ aus zwei Geraden 3 und $Z_{1}$ besteht, so sind sie als die durch diese Bezeichnung angedeuteten Elemente anzusehen, so dafs ferner die Gerade $z_{1}=\mathbf{Z}$ und der Schnitt $33_{1}=z$ ist. Die vier gemeinschaftlichen Tangenten fallen paarweise auf die Geraden $z z$ und $z z_{1}$, nämlich $(\boldsymbol{R U})=z_{z}$ und $(\boldsymbol{S} \boldsymbol{T})=z_{z_{1}}$, und daher liegen die zwei Paar Gegenecken $x$ und $x_{1}, y$ und $y_{1}$ in $z$ vereint. Eben so fallen die vier Schnitte $r, s, t, u$ parweise ( $r$ und $u, s$ und $t$ ) zusammen, in die Schnitte von $\mathbf{Z}$ mit 3 und $3_{1}$, so dafs $(r \boldsymbol{u})=\mathbf{Z}_{3}$ und $(s t)=\mathbb{Z}_{\mathfrak{Z}_{1}}$; und daher fallen die beiden Paar Gegenseiten $\mathfrak{X}$ und $\mathfrak{X}_{1}$, $\mathfrak{Y}$ und 
222 15. J. Steiner, allgem. Betrachtung üb. einand. doppelt berïhr. Kegelschnitte.

$\mathfrak{Y}_{1}$ auf $\boldsymbol{Z}$, aber trotzdem bleiben ihre Schnitte, die Pole $x$ und $y$, dennoch dadurch bestimmt, dafs sie sowohl zu $z$ und $z_{1}$, als zu den Schnitten $Z_{3}$ und $\boldsymbol{Z}_{3_{1}}$ zugeordnet harmonisch sind. Die $\boldsymbol{S}\left(\boldsymbol{C}_{z}^{2}\right)$ arten in den Strahlbüschel um $\boldsymbol{z}$ aus. Dagegen enthalten die $\boldsymbol{S}\left(\boldsymbol{C}_{x}^{2}\right)$ und $\boldsymbol{S}\left(\boldsymbol{C}_{y}^{2}\right)$ eigentlichè Curven, welche durch die Puncte $z$ und $z_{1}$ gehen und die Geraden 3 und $Z_{1}$ berühren.

III. Gestützt auf diese besondern Fälle (II.), so wie auf den obigen allgemeinen Fall ( $\$$. 10.), lassen sich folgende Aufgaben leicht behandeln und die Zahl ihrer Lösungen im Voraus bestimmen.

1) „Eine Curve $C^{2}$ zu finden, welche zwei gegebene Kegelschnitte $A^{2}$ und $B^{2}$ doppelt berührt und zudem noch entweder

$\alpha$. eine gegebene Gerade $G$ berührt; oder

ß. durch einen gegebenen Punct p geht."

Jede dieser beiden Aufgaben gestattet sechs Lösungen, und zwar bestehen die lösenden Curven aus $2 C_{x}^{2}, 2 C_{y}^{2}$ und $2 C_{z}^{2}$. Die gegenseitigen vier Schnitte des Curvenpaars $2 C_{x}^{2}$, etwa $p, p_{1}, p_{2}$ und $p_{3}$, liegen in einem der Kegelschnitte $M^{2}$ (\$.10. II. 4.), welcher durch den gegebenen Punct $p$ bestimmt ist; die drei andern Schnitte $p_{1}, p_{2}, p_{3}$ sind dadurch bestimmt, dafs einer derselben, etwa $p_{2}$, in der Geraden $x p$ liegt und dann auch die Gerade $p_{1} p_{3}$ durch $x$ geht, und zudem beide Gerade $p p_{2}$ und $p_{1} p_{3}$ zu den Seiten $\mathfrak{X}$ und $\mathfrak{X}_{1}$ zugeordnet harmonisch sind.

2) „Eine Curve $\dot{C}^{2}$ zu finden, welche eine gegebene Curve $A^{2}$ doppelt berüht und nebstdem noch entweder

a. drei gegebene Gerade 3, 31 und G berührt; oder

$\beta$. zuei gegebene Gerade 3 und $3_{1}$ berültrt und durch einen gegebenen Punct $p$ geht; oder

$\gamma$. eine gegebene Gerade $G$ berührt und durch zwei gegebene Puncte o und $z_{1}$ geht; oder endlich

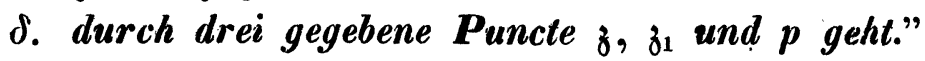

Bei jeder dieser vier Aufgaben finden', im Allgemeinen, sechs Lösungen statt; wie vorhin (1.).

3) „Eine Curve $C^{2}$ zu finden, welche entweder

$\alpha$. drei gegebene Gerade 3, 31 und $G$ berührt und durch swei gegebene Puncle z und zi geht; oder

$\beta$. zwèi gegebene Gerade 3 und 31 berührt und durch drei gegebene Puncte o, z und p geht."

Beide Mal vier Lösungen. 
15. J. St einer, allgem. Betrachtung üb. einand. doppelt berïhr. Kegelschnitte. 223

4) „Eine Curve $C^{2}$ zu finden, welche entweder

$\alpha$. vier gegebene Gerade berührt (II. 3.) und durch einen gegebenen Punct $p$ geht; oder

ß. durch vier gegebene Puncte geht (II. 4.) und eine gegebene Gerade G berührt."

Beide Mal zwei Lösungen. Und endlich:

5) „Eine Curve $C^{2}$ su finden, welche entweder

$\alpha$. funf gegebene Gerade berührt; oder

$\beta$. durch gegebene fünf Puncte geht;"

beide Mal nur eine Lösung, oder $C^{2}$ absolut bestimmt.

\$. 12.

Bernerkung. Die Aufgabe:

„Eine Curve $C^{2}$ su finden, welche drei gegebene Curven $A^{2}, B^{2}$ und $D^{2,}$ doppelt berührt,"

ist im Allgemeinen unmöglich, wie aus dem obigen (\$. 10.) leicht erhellet; sie wird erst dann möglich, wenn die gegebenen Curven eine gewisse nähere Beziehung zu einander haben, was bereits schon in der mehrerwähnten $\mathbf{A b}$ handlung (Bd.37. S. 187 d. Journ.) angegeben worden, und wovon man sich, wie folgt, leicht überzeugen kann.

Denn angenommen die Curve $C^{2}$ berühre jede der drei gegebenen Curven $\boldsymbol{A}^{2}, \boldsymbol{B}^{2}$ und $\boldsymbol{D}^{2}$ doppelt; seien etwa $\boldsymbol{A}, \boldsymbol{B}$ und $\boldsymbol{D}$ beziehlich die Berührungssehnen, und seien ferner $x, y, z ; x^{\prime}, y^{\prime}, z^{\prime} ; x^{\prime \prime}, y^{\prime \prime}, z^{\prime \prime}$ die gemeinschaftlichen Trippel conjugirte Pole, so wie $\boldsymbol{X}, \boldsymbol{Y}, \boldsymbol{Z} ; \boldsymbol{X}^{\prime}, \boldsymbol{Y}^{\prime}, \boldsymbol{Z}^{\prime}$; $\boldsymbol{X}^{\prime \prime}, \boldsymbol{Y}^{\prime \prime}, \boldsymbol{Z}^{\prime \prime}$ die gemeinschaftlichen Trippel conjugirte Polaren der Curvenpaare $\boldsymbol{A}^{2}$ und $\boldsymbol{B}^{2}, \boldsymbol{A}^{2}$ und $\boldsymbol{D}^{2}, \boldsymbol{B}^{2}$ und $\boldsymbol{D}^{2}$ : so mufs die Berührungssehne $\boldsymbol{A}$ sowohl durch einen Pol des ersten Trippels, etwa durch $x$, als auch durch einen Pol des zweiten Trippels, etwa durch $x^{\prime}$, gehen (weil $\boldsymbol{A}^{2}$ zum ersten und zweiten Curvenpaar gehört) (\$. 10. II. 1.), und dann müssen auch die Berührungssehnen $\boldsymbol{B}$ und $\boldsymbol{D}$ beziehlich durch die nämlichen Pole $x$ und $\boldsymbol{x}^{\prime}$, so wie auch beide durch einen und denselben Pol des dritten Trippels, etwa durch $x^{\prime \prime}$, gehen. Demnach müssen die drei Berührungssehnen $\boldsymbol{A}, \boldsymbol{B}$ und $\boldsymbol{D}$ allemal die Seiten eines solchen Dreiecks sein, welches irgend drei Pole, jedoch von jedem Trippel einen, zu Ecken hat, wie das Dreieck $x x^{\prime} x^{\prime \prime}$; die Combination gestattet 27 solche Dreiecke. Da nun ferner sowohl $\boldsymbol{x}$ und $\boldsymbol{X}$, als $x^{\prime}$ und $\boldsymbol{X}^{\prime}$, so wie $x^{\prime \prime}$ und $\boldsymbol{X}^{\prime \prime}$ Pol und Polare in Bezug auf die Curve $\boldsymbol{C}^{2}$ sind (\$. 10. II. 3.), so mufs das. Dreieck $x x^{\prime} x^{\prime \prime}$ mit dem Dreiseit $\boldsymbol{X} \boldsymbol{X}^{\prime} \boldsymbol{X}^{\prime \prime}$ 
224 15. J. St e iner, allgem. Betrachtung üb. einand. doppelt berühr. Kegelschnitte.

perspectivisch liegen; d. h. die drei Geraden, welche ihre Ecken in bestimmter Ordnung parweise verbinden, treffen sich in irgend einem Puncte $p$, und die drei Schnitte der entsprechenden Seitenpaare liegen in irgend einer Geraden $\boldsymbol{P}$; nämlich heifsen die den Seiten $\boldsymbol{X}, \boldsymbol{X}^{\prime}, \boldsymbol{X}^{\prime \prime}$ gegenüber liegenden Ecken des Dreiseits beziehlich $a, b, d$ (sie sind zugleich die Pole der Seiten $\boldsymbol{A}, \boldsymbol{B}, \boldsymbol{D}$, des Dreiecks $x \dot{x}^{\prime} x^{\prime \prime}$ in Bezug auf die Curve $C^{2}$ sowohl als beziehlich in Bezug auf $\boldsymbol{A}^{2}, \boldsymbol{B}^{2}, \boldsymbol{D}^{2}$ ), so treffen sich die drei Geraden $a x, b x^{\prime}, d x^{\prime \prime}$ in einem Puncte $p$, und die drei Schnitte $\boldsymbol{A} \boldsymbol{X}, \boldsymbol{B} \boldsymbol{X}^{\prime}, \boldsymbol{D} \boldsymbol{X}^{\prime \prime}$ liegen in einer Geraden $\boldsymbol{P}$ (auch sind $\boldsymbol{p}$ und $\boldsymbol{P}$ Pol und Polare in Rücksicht auf $\boldsymbol{C}^{2}$ ). $\mathrm{Zu}$ diesen Eigenschaften gesellen sich ferner noch folgende. Bezeichnet man die gegenseitigen vier Schnitte der drei, Curvenpaare durch $r, s, t, u$; $r^{\prime}, s^{\prime}, t^{\prime}, u^{\prime} ; r^{\prime \prime}, s^{\prime \prime}, t^{\prime \prime}, u^{\prime \prime}$ und ihre vier gemeinschaftlichen Tangenten durch $\boldsymbol{R}, \boldsymbol{S}, \boldsymbol{T}, \boldsymbol{U} ; \boldsymbol{R}^{\prime}, \boldsymbol{S}^{\prime}, \boldsymbol{T}^{\prime}, \boldsymbol{U}^{\prime} ; \boldsymbol{R}^{\prime \prime}, \boldsymbol{S}^{\prime \prime}, \boldsymbol{T}^{\prime \prime}, \boldsymbol{U}^{\prime \prime}$, und gleicherweise die übrigen Elemente: so gehen durch die Pole $x, x^{\prime}, \dot{x}^{\prime \prime}$ beziehlich die Seitenpaare $\mathfrak{X}$ und $\mathfrak{X}_{1}, \mathfrak{X}^{\prime}$ und $\mathfrak{X}_{1}^{\prime}, \mathfrak{X}^{\prime \prime}$ und $\mathfrak{X}_{1}^{\prime \prime}$, und in den Polaren $\boldsymbol{X}, \boldsymbol{X}^{\prime}, \boldsymbol{X}^{\prime \prime}$ liegen beziehlich die Eckenpaare $x$ und $x_{1}, x^{\prime}$ und $x_{1}^{\prime}, x^{\prime \prime}$ und $x_{1}^{\prime \prime}$; und alsdann schneiden sich von den erstern viermal drei in einem Puncte, etwa $\mathfrak{X} \mathfrak{X}^{\prime} \mathfrak{X}^{\prime \prime}$, $\mathfrak{X} \mathfrak{X}_{1}^{\prime} \mathfrak{X}_{1}^{\prime \prime}, \mathfrak{X}^{\prime} \mathfrak{X}_{1} \mathfrak{X}_{1}^{\prime \prime}$ und $\mathfrak{X}^{\prime \prime} \mathfrak{X}_{1} \mathfrak{X}_{1}^{\prime}$, und von den letztern liegen viermal drei in einer Geraden, $x x^{\prime} x^{\prime \prime}, x x_{1}^{\prime} x_{1}^{\prime \prime}, x^{\prime} x_{1} x_{1}^{\prime \prime}$ und $x^{\prime \prime} x_{1} x_{1}^{\prime}$.

Wenn also eine Curve $C^{2}$ die drei gegebenen Curven $A^{2}, B^{2}, D^{2}$ doppelt berührt, so müssen die den letztern zugehörigen Elemente, unter andern, die angegebenen Eigenschaften haben; da aber diese Eigenschaften einander bedingen, selbst von einander abhängig sind, so beschränkt sich die Bedingung für die Möglichkeit der Curve $C^{2}$ nur auf je einen Theil derselben, nämlich:

Die Curve $C^{2}$, welche die drei gegebenen Curven $A^{2}, B^{2}$ und $D^{2}$ doppelt berühren soll, ist möglich, sobald entweder:

1) von den 27 Dreiecken, welche je drei Pole, jedoch von jedem Trippel einen, zu Ecken haben, irgend eins (wie $x^{\prime} x^{\prime} x^{\prime \prime}$ ) mit dem ihm

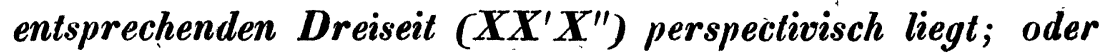

2) von den Seiten der drei Vierecke rstu, $r^{\prime} s^{\prime} t^{\prime} u^{\prime}, r^{\prime \prime} s^{\prime \prime} t^{\prime \prime} u^{\prime \prime}$ irgend drei, worunter jedoch von jedem Viereck eine (wie etwa $\mathfrak{X}, \mathfrak{X}^{\prime}, \mathfrak{X}^{\prime \prime}$ ) sich in einem Puncte $p$ treffen; oder endlich

3) von den Ecken der drei Vierseit RSTU, $R^{\prime} \boldsymbol{S}^{\prime} \boldsymbol{T}^{\prime} \boldsymbol{U}^{\prime}, \boldsymbol{R}^{\prime \prime} \boldsymbol{S}^{\prime \prime} \boldsymbol{T}^{\prime \prime} \boldsymbol{U}^{\prime \prime}$ irgend drei, unter denen jedoch von jedem Vierseit eine (urie etwa $x, x^{\prime}, x^{\prime \prime}$ ) in einer Geraden P liegen.",

Berlin, im März 1852. 